\title{
On the optimal risk allocation problem
}

\author{
Christian Burgert, Ludger Rüschendorf
}

Received: October 15, 2005; Accepted: February 20, 2006

\begin{abstract}
Summary: The optimal risk allocation problem or equivalently the problem of risk sharing is the problem to allocate a risk in an optimal way to $n$ traders endowed with risk measures $\varrho_{1}, \ldots, \varrho_{n}$. This problem has a long history in mathematical economics and insurance. In the first part of the paper we review some mathematical tools and discuss their applications to various problems on risk measures related to the allocation problem like to monotonicity properties of optimal allocations, to optimal investment problems or to an appropriate definition of the conditional value at risk. We then consider the risk allocation problem for convex risk measures $\varrho_{i}$. In general the optimal risk allocation problem is well defined only under an equilibrium condition. This condition can be characterized by the existence of a common scenario measure. We formulate a meaningful modification of the optimal risk allocation problem also for markets without assuming the equilibrium condition and characterize optimal solutions. The basic idea is to restrict the class of admissible allocations in a proper way.
\end{abstract}

\section{Introduction}

The optimal risk allocation problem or equivalently the problem of optimal risk sharing has a long history in mathematical economics and insurance and is of considerable practical and theoretical interest. In early papers the construction of linear reinsurance treaties has been based on minimizing the individual and the aggregate variance of risk (for references see Seal (1969)). Borch (1960a, 1960b, 1962) showed that based on utility functions Pareto optimal risk exchanges can be characterized and in many cases lead to familiar linear quota-sharing of the total pooled losses or to stop loss contracts and to mixtures of both. Solutions are however typically not uniquely determined which may lead to substantial fixed side payments. In several papers authors have added game theoretic considerations or additional concepts (like the concept of fairness) to arrive at a specific element in the set of Pareto optimal rules (see Borch (1960b), Lemaire (1977), Bühlmann and Jewell (1979)).

Since risk pools redistribute only actual losses and possibly the associated premiums but not the individual wealth of the company it is natural to include side constraints in the exchange protocol of the form $Y_{i} \geq A_{i}$ for the components $Y_{i}$ of the allocation and some constant or random bounds $A_{i}$, to limit negative charges or payouts of company $i$. 
Similarly also upper constraints of the form $Y_{i} \leq A_{i}+B_{i}$ have been introduced to protect the liquidity of the individual companies. The importance of side constraints has been suggested by Borch (1968) and has formally been introduced and applied in Gerber (1978, 1979).

Several authors have extended the framework as e.g. to include more general utilities, to consider incomplete preferences, or to include the presence of background risk. The allocation problem has also been considered in the context of financial risks. For some of the large number of references see Leland (1980), Chavallier and Müller (1994), and Barrieu and El Karoui (2002a, 2002b, 2003, 2004, 2005), Dana and Scarsini (2005), Chateauneuf, Dana, and Tallon (2000), Gerber (1979), Landsberger and Meilijson (1994), Denault (2001), Dana and Meilijson (2003), Heath and Ku (2004), Carlier and Dana (2003), Burgert and Rüschendorf (2005), Jouini, Schachermayer, and Touzi (2005), Dana (2005) and references therein. Also more general types of risk measures (distortion type, coherent, convex, comonotone risk measures) have been considered for the allocation problem. For the background literature on risk measures and their applications to finance and insurance we refer to Kaas, Goovaerts, Dhaene, and Denuit (2001), Delbaen (2002), and Föllmer and Schied (2004).

In our paper which is based on these developments we consider the following formulation of the optimal risk allocation problem. Let $(\Omega, \mathcal{A}, P)$ be a nonatomic probability space and consider a market whith $n$ traders endowed with convex risk measures $\varrho_{1}, \ldots, \varrho_{n}$. The problem is to characterize optimal allocations of a risk $X \in L^{\infty}(P)$ to the $n$ traders, i.e. to determine solutions of the problem

$$
\sum_{i=1}^{n} \varrho_{i}\left(X_{i}\right)=\inf !
$$

under all allocations of $X$ to the traders, i.e. under all decompositions $X=\sum_{i=1}^{n} X_{i}$, $X_{i} \in L^{\infty}(P)$. (The same problem can also be considered in $L^{p}(P)$ for any $1 \leq p \leq \infty$.) Solutions of the risk allocation problem are not unique but in fact are given under an equilibrium condition by the set of all Pareto optimal allocations, as follows from a general separation argument and the translation invariance of the $\varrho_{i}$ (see Gerber (1979, pp. 8896). Thus the optimal allocation problem can be interpreted as problem to minimize the total risk of a risk sharing contract but also as a basic tool to determine Pareto optimal allocations. The value of the optimal allocation problem is given by the infimal convolution $\widehat{\varrho}=\varrho_{1} \wedge \cdots \wedge \varrho_{n}$ defined by

$$
\widehat{\varrho}(X)=\inf \left\{\sum_{i=1}^{n} \varrho_{i}\left(X_{i}\right) ; \quad X_{i} \in L^{\infty}(P), \sum_{i=1}^{n} X_{i}=X\right\}
$$

for $X \in L^{\infty}(P)$ (or more generally $X \in L^{p}(P), 1 \leq p \leq \infty$ ).

In the first part of the paper we show that the general formulation of the optimal risk allocation problem in (1.1) (1.2) makes only sense under a Pareto equilibrium condition (E). In vague form (E) can be stated as follows. A market is in equilibrium if in a balance of supply and demand it is not possible to lower the risk of some traders without increasing that of some other traders. This equilibrium condition (E) has been characterized for coherent risk measures $\varrho_{1}, \ldots, \varrho_{n}$ in Heath and $\mathrm{Ku}(2004)$ and in Burgert and 
Rüschendorf (2005) in terms of the scenario measures of the $\varrho_{i}$. We give an extension of this characterization to convex risk measures and establish that $\widehat{\varrho}$ is a convex risk measure if and only if the Pareto equilibrium condition (E) holds true.

The main new part of this paper is concerned with the allocation problem in the case that the equilibrium condition does not hold. In this case the above formulation of the optimal allocation problem leads to inconsistencies. We introduce a new class of restrictions on the set of allocations which we call admissible allocations and consider the problem of optimal allocations with respect to this restricted class. In comparison to the constraints as dealt with in Gerber (1978) we postulate constraints on the compensation structure of the allocation $X_{i}$ motivated as above to limit negative charges or payouts and to protect the liquidity. The bounds depend on the absolute size of the total risk $X$. From a mathematical point of view our side constraints are connected with a similar idea in portfolio theory, where one considers (lower bounded) admissible strategies in order to exclude strategies which allow arbitrage. As consequence we obtain a new convex risk measure - called the convex admissible infimal convolution risk measure - which describes the optimal total admissible risk $\sum_{i=1}^{n} \varrho_{i}\left(X_{i}\right)$ in the market.

The risk sharing problem is a problem where the traders minimize the total risk by some kind of exchange contracts. This can be considered as an optimistic attitude towards risk. It aims to construct an optimal admissible exchange which is typical for insurance and reinsurance contracts. In the final part of our paper we consider the opposite view from the perspective of a regulatory agent in a financial market who takes care that the individual agents (traders) have enough capital reserves to cover their part of the risk $X_{i}$ in any allocation $X=\sum_{i=1}^{n} X_{i}$ to the $n$ traders. The regulatory agent considers any possible (admissible) allocation and determines the total risk in the worst case which is the necessary total capital reserve. Therefore, we describe this situation as a situation with a cautious risk attitude. Again as a result we obtain a new coherent risk measure describing the worst case total admissible risk.

In Section 2 of this paper we review some mathematical tools and in particular discuss the relevance of the generalized distribution transform as well as the Hoeffding-Fréchet bounds and the comonotone improvement result for various risk measure problems. These are in particular useful tools for establishing natural monotonicity properties of optimal allocations. They also simplify arguments for optimal investment problems and lead in a natural way to a correct definition of the conditional value at risk measure. In Section 3 we then consider the optimal allocation problem which corresponds to an optimistic risk attitude. In Section 4 we discuss the allocation problem under a cautious risk attitude, i.e. under the view of a regulatory agent. In both cases as a result we obtain convex risk measures which are relevant and correspond to the risk attitude.

\section{Generalized distribution transform, Hoeffding-Fré- chet bounds, and the comonotone improvement result}

This section deals with some mathematical tools which are useful for deriving monotonicity properties of optimal allocations but also are useful for various further problems 
on risk measures. We assume throughout this paper that the underlying probability space $(\Omega, \mathcal{A}, P)$ is nonatomic.

For any real random variable $X$ on $\Omega$ let for $x \in \mathbb{R}$ and $\lambda \in[0,1]$

$$
F(x, \lambda):=P(X<x)+\lambda P(X=x)
$$

be the modified distribution function. For a random variable $V$ uniformly distributed on $(0,1)(V \sim U(0,1))$ and independent of $X$ (which we assume to exist on $(\Omega, \mathcal{A}, P))$ we define the (generalized) distribution transform of $X$ by

$$
U:=F(X, V) \text {. }
$$

Then it is not difficult to check that

$$
U \sim U(0,1)
$$

and

$$
X=F_{X}^{-1}(U)[P]
$$

The generalized distribution transform in (2.2) can be found in Ferguson (1967, Lemma 1, p. 216). An extension of this transform to multivariate random vectors is in Rüschendorf (1981). (2.2), (2.4) give an explicit construction for a result of Ryff (1970), which states the existence of some $U \sim U(0,1)$ with (2.4). We will give some examples of the usefulness of this distribution transform.

A second tool of considerable importance are the Hoeffding-Fréchet bounds (holding true also without assuming $(\Omega, \mathcal{A}, P)$ to be nonatomic): For any $X, Y$ such that $X \cdot Y \in$ $L^{1}(P)$ and any $U \sim U(0,1)$ holds

\section{Hoeffding-Fréchet bounds}

$$
E F_{X}^{-1}(1-U) F_{Y}^{-1}(U) \leq E X Y \leq E F_{X}^{-1}(U) F_{Y}^{-1}(U) .
$$

Remark 2.1 Inequality (2.5) is due to Hoeffding (1940). The related bounds for the joint distribution functions are given independently at the same time by Fréchet (1940). Using the above mentioned result of Ryff (1970) the Hoeffding-Fréchet bounds (2.5) also follow from rearrangement inequalities for real functions on $[0,1]$ due to Hardy, Littlewood, and Pólya (1952). The literature on rearrangement inequalities in function spaces making this connection to random variables however came up only in the 70's and, therefore, it seems appropriate to assign this fundamental inequality to Hoeffding and Fréchet.

A third important tool in connection with the allocation problem is the following comonotone improvement result of Landsberger and Meilijson (1994): Let $X_{1}, \ldots, X_{n} \in$ $L^{1}(P)$ and $X:=\sum_{i=1}^{n} X_{i}$, then there exist $X_{i}^{*} \leq_{\mathrm{cx}} X_{i}$, such that

$$
X=\sum_{i=1}^{n} X_{i}^{*} \text { and } X_{1}^{*}, \ldots, X_{n}^{*} \text { are comonotone. }
$$


Here $\leq_{\mathrm{cx}}$ denotes the convex stochastic ordering and $\left(X_{1}^{*}, \ldots, X_{n}^{*}\right)$ are called comonotone if for all $i \neq j$

$$
\left(X_{i}^{*}(w)-X_{i}^{*}\left(w^{\prime}\right)\right)\left(X_{j}^{*}(w)-X_{j}^{*}\left(w^{\prime}\right)\right) \geq 0[P \otimes P]
$$

i.e. all components are similarly ordered. Comonotonicity of $\left(X_{i}^{*}\right)$ is known to be equivalent to the existence of nondecreasing functions $f_{i}$, such that $X_{i}^{*}=f_{i}\left(X^{*}\right), 1 \leq i \leq n$, for $X^{*}=\sum_{i=1}^{n} X_{i}^{*}=\sum_{i=1}^{n} X_{i}=X$.

A risk measure is called consistent w.r.t. $\leq_{\mathrm{cx}}$, if it is monotone w.r.t. the partial convex order $\leq_{\mathrm{cx}}$. Consistent risk measures are law invariant. Consistency of a risk measure $\varrho$ w.r.t. convex ordering is close to convexity of $\varrho$.

Proposition 2.2 Let $\varrho$ be a law invariant risk measure on $L^{\infty}(P)$.

a) If $\varrho$ is a convex risk measure then $\varrho$ is $\leq_{c x}$-consistent.

b) If $\varrho$ is convex on any class of comonotone risks and $\leq_{c x}$-consistent, then $\varrho$ is a convex risk measure.

Proof: For a) see Föllmer and Schied (2004), Burgert and Rüschendorf (2004), and Dana (2005).

b) For any $X, Y \in L^{\infty}(P)$ and $\alpha \in(0,1)$ holds $\alpha X+(1-\alpha) Y \leq_{\mathrm{cx}} \alpha F_{X}^{-1}(U)$ $+(1-\alpha) F_{Y}^{-1}(U)$. Therefore, using convexity of $\varrho$ on sets of comonotone risks and $\leq_{\mathrm{cx}}$-consistency of $\varrho$ we obtain $\varrho(\alpha X+(1-\alpha) Y) \leq \alpha \varrho(X)+(1-\alpha) \varrho(Y)$.

In case $\varrho$ is comonotone additive and coherent part b) follows from Wang, Young, and Panjer (1997). As consequence of the comonotone improvement result we thus obtain

Proposition 2.3 (Monotonicity result) Let $\varrho_{i}$ be convex, law invariant risk measures and let $\left(X_{i}\right)$ be an allocation of $X$ in $L^{\infty}(P)$, then there exists an allocation $\left(X_{i}^{*}\right)$ of $X$ with $\varrho_{i}\left(X_{i}^{*}\right) \leq \varrho_{i}\left(X_{i}\right), 1 \leq i \leq n$, and $X_{i}^{*}=f_{i}(X)$ for some monotonically nondecreasing functions $f_{i}$.

It is obvious that this result also holds true for risk measures on $L^{p}(P), 1 \leq p \leq \infty$.

The monotone improvement result implies the existence of optimal allocations $\left(X_{i}^{*}\right)$, such that $X_{i}^{*}=f_{i}(X), f_{i}$ nondecreasing (see Carlier and Dana (2003), Jouini, Schachermayer, and Touzi (2005, Theorem 6)) provided the infimum over all allocations is finite, $\varrho_{i}$ are convex law invariant and $X \in L^{\infty}(P)$.

The same monotone structure of optimal solutions remains true if we add an individual rationality constraint to the problem, i.e.: Let $X=\sum_{i=1}^{n} X_{i}$ be an allocation of $X$ and consider the problem

$$
\text { (AR) } \quad \begin{cases}\sum_{i=1}^{n} \varrho_{i}\left(Y_{i}\right) & =\min ! \\ \sum Y_{i} & =X \\ Y_{i} & \leq_{\mathrm{cx}} X_{i} .\end{cases}
$$


Again a solution $\left(X_{i}^{*}\right)$ exists such that $X_{i}^{*}=f_{i}(X), f_{i}$ nondecreasing. The additional condition $Y_{i} \leq_{\mathrm{cx}} X_{i}$ is motivated by the argument, that trader $i$ will only accept the new risk $Y_{i}$ in exchange to his risk $X_{i}$ if $Y_{i}$ is preferable to $X_{i}$ for trader $i$, i.e., $Y_{i}$ has a smaller risk than $X_{i}$.

We discuss briefly some further interesting applications of these tools relevant in risk theory.

\section{Copulas}

Let $X=\left(X_{1}, \ldots, X_{d}\right)$ be a $d$-dimensional random vector with marginal d.f.s $F_{i}=F_{X_{i}}$, $1 \leq i \leq d$. Then applying the generalized distribution transform to the components $U_{i}:=F_{i}\left(X_{i}, V\right)$ we obtain a random vector

$$
U=\left(U_{1}, \ldots, U_{d}\right)
$$

with uniform marginals. Further,

$$
X_{i}=F_{i}^{-1}\left(U_{i}\right)[P]
$$

and the d.f. $F_{X}$ of $X$ is given by

$$
F_{X}(x)=F_{U}\left(F_{1}\left(x_{1}\right), \ldots, F_{d}\left(x_{d}\right)\right) .
$$

Thus $U$ is a copula of $X$.

The generalized distribution transform allows a simple construction of the copula which is an important tool for the analysis of the dependence structure of risk vectors. Thus Sklar's theorem is a direct consequence of the generalized distribution transform. For the application of the distributional transform to obtain bounds for risk functionals of portfolio vectors see the survey paper Rüschendorf (2005).

\section{Conditional value at risk}

Let $x_{\alpha}=g_{\alpha}(X)=\inf \{x: P(X \leq x) \geq \alpha\}$ denote the lower $\alpha$-quantile of the risk $X$. It is well known that the conditional tail expectation

$$
\operatorname{TCE}_{\alpha}(X):=-E\left(X \mid X \leq x_{(\alpha)}\right)
$$

in general does not define a coherent risk measure except when restricted to continuous distributions. From the point of view of the generalized distributional transform it is natural to define the conditional value at risk by

$$
\operatorname{CVR}_{\alpha}(X):=-E(X \mid U \leq \alpha),
$$

where $U=F(X, V) \sim U(0,1)$ is the distribution transform of $X$. With this definition we obtain

$$
\begin{aligned}
\operatorname{CVR}_{\alpha}(X) & =-\frac{1}{\alpha} E X 1(U \leq \alpha) \\
& =-\frac{1}{\alpha}\left[E\left(X 1\left(X<x_{(\alpha)}\right)+x_{(\alpha)} 1\left(X=x_{(\alpha)}, U \leq \alpha\right)\right)\right]
\end{aligned}
$$




$$
\begin{aligned}
& =-\frac{1}{\alpha}\left[E X 1\left(X<x_{(\alpha)}\right)\right. \\
& \left.+x_{(\alpha)} 1\left(X=x_{(\alpha)}, P\left(X<x_{(\alpha)}\right)+V P\left(X=x_{(\alpha)}\right) \leq \alpha\right)\right] \\
& =-\frac{1}{\alpha}\left[E X 1\left(X<x_{(\alpha)}\right)+x_{(\alpha)} P\left(X=x_{(\alpha)}\right) 1\left(V \leq \frac{\alpha-P\left(X<x_{(\alpha)}\right)}{P\left(X=x_{(\alpha)}\right)}\right)\right] \\
& =-\frac{1}{\alpha}\left[E X 1\left(X<x_{(\alpha)}\right)+x_{(\alpha)}\left(\alpha-P\left(X<x_{(\alpha)}\right)\right)\right] \\
& =\mathrm{ES}_{\alpha}(X) \text {. }
\end{aligned}
$$

The modified definition of conditional value at risk is identical to the expected shortfall $\mathrm{ES}_{\alpha}(X)$; it therefore is also identical to the average value at risk $\operatorname{AVaR}_{\alpha}(X)$, and thus is a coherent risk measure. As a result the definition of conditional value at risk in (2.13) appears to be appropriate and leads to a coherent risk measure.

\section{Optimal investment problem}

The following optimal investment problem is a classical problem in portfolio theory (see Dana (2005) with many references on this problem).

For $X^{\prime} \in L^{1}(\Omega), X \in L^{\infty}(\Omega)$ consider

$$
(\mathcal{E}) \quad\left\{\begin{array}{l}
E X^{\prime} C=\min ! \\
C \leq_{\mathrm{cx}} X \\
C \in L^{\infty}(P) .
\end{array}\right.
$$

Considering $X^{\prime}$ as a price density the problem is to find under all investments $C$ which are more attractive than $X$ one with the lowest price. The solution $e\left(X, X^{\prime}\right)$ of $(\mathcal{E})$ is called reservation price of $X$ (see Jouini and Kallal (2000)). Its value is given by

$$
e\left(X, X^{\prime}\right)=\int_{0}^{1} F_{X^{\prime}}^{-1}(1-t) F_{X}^{-1}(t) d t
$$

(see also Dana (2005, Theorem 1)).

The proof of the following lemma, which is crucial for this problem, is simplified by the general distribution transform.

\section{Lemma 2.4 For any r.v.s $X, Y$ holds}

$$
\inf \left\{E X Y^{\prime} ; Y^{\prime} \sim Y\right\}=e(X, Y)=\int_{0}^{1} F_{X}^{-1}(t) F_{Y}^{-1}(1-t) d t .
$$

Proof: By the Hoeffding-Fréchet inequality the r.h.s of (2.16) is a lower bound. Conversely, let $V \sim U(0,1)$ be independent of $X$ and define $U:=F_{X}(X, V)$. Then $F_{X}^{-1}(U)=$ $X[P]$ and the r.h.s is attained by the admissible pair $=\left(F_{X}^{-1}(U), F_{Y}^{-1}(1-U)\right)=$ $\left(X, F_{Y}^{-1}(1-U)\right)$. 
Now (2.15) follows from the monotonicity of $e\left(\cdot, X^{\prime}\right)$ with respect to $\leq_{\mathrm{cx}}$, which is a well known result in stochastic ordering. Note that the optimal investment $C^{*}$ is given by $F_{Y}^{-1}(1-U)=g(X, V) . C^{*}$ is a decreasing function of $X$ and $V$. An equivalent investment is $\bar{C}=E\left(C^{*} \mid X\right)=\int_{0}^{1} g(X, v) d v=: \varphi(X)$ which is decreasing in $X$.

\section{Minimal liability risk problem}

The following minimal liability risk problem of Schied (2004) is similar to the optimal investment problem in III:

$$
\text { (L) }\left\{\begin{aligned}
\varrho(-X) & =\text { inf ! } \\
E \varphi X & \geq v
\end{aligned}\right.
$$

where $\varrho$ is a law invariant risk measure and $\varphi$ is a pricing density $0 \leq \varphi, E_{P} \varphi=1$. The aim is to minimize the risk of the liability $-X$ under all $X$ with price of $-X$ smaller than $-v$. There exists a solution $X^{*}$ of $(L)$ as shown in Schied (2004) if $\varrho$ is lower semicontinuous.

Proposition 2.5 (Monotone solutions of the liability problem) a) There exists a solution $X^{*}$ of the minimal liability problem $(L)$ such that $\left(X^{*}, \varphi\right)$ are comonotone.

b) If $\varrho$ is a convex, law invariant risk measure, then there exists a solution $X^{*}$ of the form $X^{*}=g(\varphi)$ with $g$ nondecreasing.

\section{Proof:}

a) Let $V \sim U(0,1)$ be independent of $X, \varphi$ and let $U_{\varphi}:=F_{\varphi}(\varphi, V) \sim U(0,1)$ be the generalized distributional transform and $X^{*}:=F_{\widetilde{X}}^{-1}\left(U_{\varphi}\right)$, where $\widetilde{X}$ is a solution of (2.17). Then $\tilde{X} \sim X^{*}$ and thus $\varrho(-\tilde{X})=\varrho\left(-X^{*}\right)$. Further, using $F_{\varphi}^{-1}\left(U_{\varphi}\right)=\varphi[P]$, we obtain from the Hoeffding-Fréchet inequality $v \leq E \varphi \widetilde{X} \leq E F_{\varphi}^{-1}\left(U_{\varphi}\right) F_{\widetilde{X}}^{-1}\left(U_{\varphi}\right)=$ $E \varphi X^{*}$. Thus also $X^{*}$ is a solution and $X^{*}=h(\varphi, V)$, where $h$ is monotonically nondecreasing in $\varphi, V$. Thus $X^{*}, \varphi$ are comonotone.

b) If $\varrho$ is a convex law invariant risk measure, and if we define

$$
\bar{X}:=E\left(X^{*} \mid \varphi\right)=\int h(\varphi, v) d P^{V}(v)=\int h(\varphi, v) d v=g(\varphi), g \uparrow
$$

then we obtain $E \varphi \bar{X}=E \varphi X^{*} \geq v$ and $\varrho(-\bar{X}) \leq \varrho\left(-X^{*}\right)=\varrho(-\tilde{X})$, since $\varrho$ is $\leq_{\mathrm{cX}}$-consistent. Thus we obtain a solution which is a monotonically nondecreasing function of $\varphi$.

\section{Optimal allocation of risks with optimistic risk attitude}

In this and in the following section we assume that $\varrho_{i}$ are convex risk measures on $L^{\infty}(P)$, i.e. monotone, translation invariant convex functionals on $L^{\infty}(P)$. The acceptance set $\mathcal{A}=\mathcal{A}_{\varrho}$ of a convex risk measure $\varrho$ is given by the convex set

$$
\mathcal{A}_{\varrho}=\left\{X \in L^{\infty}(P) ; \varrho(X) \leq 0\right\} .
$$


Convex risk measures are characterized by a representation of the form

$$
\varrho(X)=\sup _{Q \in \operatorname{ba}(P)} E_{Q}(-X-\alpha(Q)), \quad \forall X \in L^{\infty}(P),
$$

where $\mathrm{ba}(P)$ is the set of finitely additive $P$-continuous measures and $\alpha: \mathrm{ba}(P) \rightarrow$ $(-\infty, \infty]$ is a convex penalty function, which can be chosen as Fenchel-Legendre conjugate of $\varrho$

$$
\alpha(Q)=\sup _{X \in L^{\infty}(P)}\left(E_{Q}(-X)-\varrho(X)\right)
$$

(see Föllmer and Schied (2004)). Further the sup can be restricted to the class $\mathcal{M}^{1}(P)$ of probability measures absolutely continuous w.r.t. $P$ if the acceptance set

$$
\mathcal{A}_{\varrho}=\left\{X \in L^{\infty}(P) ; \varrho(X) \leq 0\right\} \text { is } \sigma\left(L^{\infty}(P), L^{1}(P)\right) \text { closed, }
$$

or equivalently if $\varrho$ is Fatou continuous (i.e. $\left|X_{n}\right| \leq K, \forall n$ and $X_{n} \stackrel{P}{\rightarrow} X$ implies $\left.\varrho(X) \leq \underline{\lim } \varrho\left(X_{n}\right)\right)$ resp., if $\varrho$ is lower semicontinuous w.r.t. $\sigma\left(L^{\infty}(P), L^{1}(P)\right)$.

We will introduce a meaningful general formulation of the optimal allocation problem and derive some properties of optimal allocations. It was observed in the case of coherent risk measures $\varrho_{i}$ in Heath and $\mathrm{Ku}$ (2004) and Burgert and Rüschendorf (2005) that the optimal allocation problem as in (2.1) is well defined only under an additional Pareto equilibrium condition. This observation extends to the case of convex risk measures $\varrho_{i}$ with penalty functions $\alpha_{i}$ as in (3.3).

Definition 3.1 (Pareto equilibrium) A market model with risk $\varrho_{1}, \ldots, \varrho_{n}$ is in Pareto equilibrium, if

$$
\begin{aligned}
& X_{i} \in L^{\infty}(P) \text { with } \sum_{i=1}^{n} X_{i}=0 \text { and } \varrho_{i}\left(X_{i}\right) \leq \varrho_{i}(0), \quad 1 \leq i \leq n, \\
& \text { implies } \varrho_{i}\left(X_{i}\right)=\varrho_{i}(0), \quad 1 \leq i \leq n
\end{aligned}
$$

In a balance of supply and demand the market is in Pareto equilibrium not possible to lower the risk of some traders without increasing that of others. Vaguely one could say that there is no arbitrage situation concerning risk. There is a seemingly stronger version of the equilibrium condition:

(SE)

\section{Strong equilibrium}

$$
\text { If } X_{i} \in L^{\infty}(P) \text { with } \sum_{i=1}^{n} X_{i}=0 \text {, then } \sum_{i=1}^{n} \varrho_{i}\left(X_{i}\right) \geq \sum_{i=1}^{n} \varrho_{i}(0) \text {. }
$$

It is immediate to see that $(\mathrm{SE}) \Rightarrow(\mathrm{E})$, but in fact both conditions are equivalent. 
Proposition 3.2 The equilibria conditions (E) and (SE) are equivalent.

Proof: Assume that for some $X_{i} \in L^{\infty}(P)$ with $\sum_{i=1}^{n} X_{i}=0$ holds $\sum_{i=1}^{n}\left(\varrho_{i}\left(X_{i}\right)-\right.$ $\left.\varrho_{i}(0)\right)=: c<0$. Then with $c_{i}:=\varrho_{i}\left(X_{i}\right)-\varrho_{i}(0)$ and $Z_{i}:=X_{i}+c_{i}-\frac{c}{n}$ holds: $\sum_{i=1}^{n} Z_{i}=0$ and

$$
\varrho_{i}\left(Z_{i}\right)=\varrho_{i}\left(X_{i}\right)-c_{i}+\frac{c}{n}=\varrho_{i}(0)+\frac{c}{n}<\varrho_{i}(0), \quad 1 \leq i \leq n .
$$

Thus we obtain a contradiction to $(\mathrm{E})$.

Thus under the Pareto equilibrium condition (E) the sum of all risks in a balance situation is greater than the cumulative risk of zero. The infimal convolution $\widehat{\varrho}=\varrho_{1} \wedge$ $\cdots \wedge \varrho_{n}$ as defined in (1.2) describes the optimal allocation of risk. $\widehat{\varrho}$ itself is, as a functional of $X$, a risk measure.

If $\widehat{\varrho}$ is a convex risk measure, then by Theorem 3.6 in Barrieu and El Karoui (2003) the penalty function $\alpha \widehat{\varrho}$ of $\widehat{\varrho}$ is given by

$$
\alpha_{\widehat{\varrho}}=\sum_{i=1}^{n} \alpha_{i}
$$

A characterization of Pareto equilibrium is given in the following proposition in terms of the penalty functions $\alpha_{i}$.

Proposition 3.3 Let $\mathcal{P}_{i}:=\left\{Q \in \mathrm{ba}(P): \alpha_{i}(Q)<\infty\right\}$ denote the 'scenario sets' of $\varrho_{i}$, $1 \leq i \leq n$. Then the following conditions are equivalent.

1) $\widehat{\varrho}$ is a convex risk measure.

2) $\widehat{\varrho}(0)>-\infty$.

3) $\widehat{\varrho}(0)=\sum_{i=1}^{n} \varrho_{i}(0)$.

4) (SE) holds.

5) $\bigcap_{i=1}^{n} \mathcal{P}_{i} \neq \emptyset$.

Proof: The first equivalence is obvious. The equivalence of 1) and 3) follows from the fact that the penalty function $\alpha \widehat{\varrho}$ of $\widehat{\varrho}$ is given by

$$
\alpha_{\widehat{\varrho}}=\sum_{i=1}^{n} \alpha_{i}
$$

The equivalence of 3) and 4) follows from the definition of $\widehat{\varrho}$ since

$$
\widehat{\varrho}(0)=\inf \left\{\sum_{i=1}^{n} \varrho_{i}\left(X_{i}\right) ; \sum_{i=1}^{n} X_{i}=0\right\}=\sum_{i=1}^{n} \varrho_{i}(0)
$$


is equivalent to

$$
\sum_{i=1}^{n} X_{i}=0 \Rightarrow \sum_{i=1}^{n} \varrho_{i}\left(X_{i}\right) \geq \sum_{i=1}^{n} \varrho_{i}(0)
$$

i.e. to condition (SE).

The equivalence of 2) and 5) is obvious.

Remark 3.4 In the case of coherent risk measures $\varrho_{i}, \alpha_{i}(Q) \in\{0, \infty\}$ for all $i$ and the sets $\mathcal{P}_{i}=\left\{Q \in \mathrm{ba}(P), \alpha_{i}(Q)=0\right\}$ defined in Proposition 3.3 are identical with the sets of scenario measures of $\varrho_{i}$. Thus Proposition 3.3 implies the characterization of Heath and $\mathrm{Ku}$ (2004) resp. Burgert and Rüschendorf (2005) in this case. The equilibrium condition (E) is equivalent to the condition $\bigcap_{i=1}^{n} \mathcal{P}_{i} \neq \emptyset$, i.e., there exists a scenario measure $Q$ which is shared by all traders in the market.

In the case that the Pareto equilibrium condition does not hold we introduce the following formulation of the optimal allocation problem which makes sense also without assuming condition $(\mathrm{E})$.

Define a decomposition $X=\sum_{i=1}^{n} X_{i}$ to be admissible if $X(\omega) \geq 0$ implies that $0 \leq X_{i}(\omega)$ and $X(\omega) \leq 0$ implies that $X_{i}(\omega) \leq 0$. Let $A(X)$ denote the set of admissible decompositions $\left(X_{i}\right)$ of $X$. We define the admissible infimal convolution $\varrho_{*}$ as the value of the allocation problem restricted to admissible allocations

$$
\varrho_{*}(X):=\inf \left\{\sum_{i=1}^{n} \varrho_{i}\left(X_{i}\right) ; \quad\left(X_{i}\right) \in A(X)\right\} .
$$

Obviously, we have

$$
\varrho_{*}(X) \leq \min \left(\varrho_{i}(X)-\varrho_{i}(0)\right)+\sum_{i=1}^{n} \varrho_{i}(0) .
$$

The restriction to admissible decompositions of risks excludes, similarly as the restriction to admissible strategies in portfolio theory, that effects like doubling strategies paradoxes may occur in risk allocation.

In the following theorem we derive a representation of $\varrho_{*}$ in terms of the representation scenarios $\mathcal{P}_{i}:=\left\{Q \in \operatorname{ba}(P) ; \quad \alpha_{i}(Q)<\infty\right\}$ of $\varrho_{i}$. For $P_{i} \in \mathcal{P}_{i}$ define by $P_{1} \wedge \cdots \wedge P_{n}$ the lattice infimum of $\left(P_{i}\right)$ in the lattice ba $(P)$ and by $P_{1} \vee \cdots \vee P_{n}$ the lattice supremum of $\left(P_{i}\right)$ in ba $(P)$ (see Rao and Rao (1983, Theorem 2.2.1)). If $P_{i}$ are probability measures with densities $f_{i}$ w.r.t. $\mu$ then $P_{1} \wedge \cdots \wedge P_{n}$ resp. $P_{1} \vee \cdots \vee P_{n}$ have densities $\min \left\{f_{i}\right\}$ resp. $\max \left\{f_{i}\right\}$ w.r.t. $\mu$.

Theorem 3.5 Let $\varrho_{j}$ be convex risk measures with penalty functions $\alpha_{j}$. The admissible infimal convolution $\varrho_{*}$ is given by

$$
\varrho_{*}(X)=\sup \left\{\int X_{-} d \bigwedge_{j} P_{j}-\int X_{+} d \bigvee_{j} P_{j}-\sum_{j} \alpha_{j}\left(P_{j}\right) ; \quad P_{j} \in \mathcal{P}_{j}, 1 \leq j \leq n\right\} .
$$


Proof: The proof is similar to that for coherent risk measures in Burgert and Rüschendorf (2005). The class of admissible decompositions has an alternative description like in multiple decision problems:

$$
A(X)=\left\{\left(\varphi_{i} X\right) ; \quad 0 \leq \varphi_{i} \leq 1, \sum_{i=1}^{n} \varphi_{i}=1\right\}
$$

thus we consider decompositions of the form $\left(\varphi_{i} X\right)$.

For $P_{i} \in \mathcal{P}_{i}, 1 \leq i \leq n$ and $Y:=-X \in L^{\infty}(P)$ holds

$$
a_{P_{1}, \ldots, P_{n}}(Y):=\inf \left\{\sum_{i=1}^{n} \int \varphi_{i} Y d P_{i} ; \quad 0 \leq \varphi_{i}, \sum_{i=1}^{n} \varphi_{i}=1\right\}
$$

has a solution $\left(\varphi_{i}^{*}\right)$ and if $Y(\omega)>0$, then $\left\{\varphi_{i}^{*}>0\right\} \subset\left\{P_{i}=\bigwedge_{j=1}^{n} P_{j}\right\}$ and if $Y(\omega)<0$, then $\left\{\varphi_{i}^{*}>0\right\} \subset\left\{P_{i}=\bigvee_{j=1}^{n} P_{j}\right\}$. Thus

$$
\begin{aligned}
a_{P_{1}, \ldots, P_{n}}(Y) & =\int_{Y \geq 0} Y d \bigwedge_{j=1}^{n} P_{j}+\int_{Y<0} Y d \bigvee_{j=1}^{n} P_{j} \\
& =\int Y_{+} d \bigwedge P_{j}-\int Y_{-} d \bigvee P_{j} \\
& =\int X_{-} d \wedge P_{j}-\int X_{+} d \bigvee P_{j}
\end{aligned}
$$

Therefore, we obtain

$$
\begin{aligned}
\varrho_{*}(X)=\inf _{\left(\varphi_{i}\right)} \sum_{i} \varrho_{i}\left(\varphi_{i} X\right) & =\inf _{\left(\varphi_{i}\right)} \sum_{i} \sup _{P_{i} \in \mathcal{P}_{i}}\left(\int\left(-\varphi_{i} X\right) d P_{i}-\alpha_{i}\left(P_{i}\right)\right) \\
& =\inf _{\left(\varphi_{i}\right)}\left[-\sum_{i=1}^{n} \inf _{P_{i} \in \mathcal{P}_{i}}\left(\int \varphi_{i} X d P_{i}+\alpha_{i}\left(P_{i}\right)\right)\right] \\
& =-\sup _{\left(\varphi_{i}\right)} \sum_{i} \inf _{P_{i} \in \mathcal{P}_{i}}\left(\int \varphi_{i} X d P_{i}+\alpha_{i}\left(P_{i}\right)\right) .
\end{aligned}
$$

We now apply the minimax theorem for games $(A, B, f)$ of concave-convex type, (see Müller (1971)). Let $f: A \times B \rightarrow \overline{\mathbb{R}}, A, B \neq \emptyset$ and assume that

1) $\forall b_{1}, b_{2} \in B, \alpha \in[0,1]$ there exists a $b \in B$ such that for all $a \in A$ holds

$$
f(a, b) \leq(1-\alpha) f\left(a, b_{1}\right)+\alpha f\left(a, b_{2}\right) .
$$

2) $\forall a_{1}, a_{2} \in A, \alpha \in[0,1]$ there exists an $a \in A$ such that for all $b \in B$ holds

$$
f(a, b) \geq(1-\alpha) f\left(a_{1}, b\right)+\alpha f\left(a_{2}, b\right)
$$


If $f<\infty$ and if for some topology $\tau$ on $A$ holds $A$ is $\tau$-compact and $\forall b \in B$, $f(\cdot, b): A \rightarrow \mathbb{R}$ is upper semicontinuous, then

$$
\inf _{b \in B} \sup _{a \in A} f(a, b)=\sup _{a \in A} \inf _{b \in B} f(a, b) .
$$

We choose $A=\left\{\left(\varphi_{i}\right) ; 0 \leq \varphi_{i}, \sum \varphi_{i}=1\right\}$, which is compact in weak*-topology, $B=\mathcal{P}_{1} \times \cdots \times \mathcal{P}_{n}$ and $f\left(\left(\varphi_{i}\right),\left(P_{i}\right)\right)=\sum_{i=1}^{n}\left(\int \varphi_{i} X d P_{i}+\alpha_{i}\left(P_{i}\right)\right)$. By linearity of $f$ and convexity of $\mathcal{P}_{i}$ and $A$ the conditions of the minimax theorem are fulfilled and we obtain from the first part of the proof

$$
\begin{aligned}
\varrho_{*}(X) & =-\inf _{P_{i} \in \mathcal{P}_{i}} \sup _{\left(\varphi_{i}\right)} \sum_{i}\left(\int \varphi_{i} X d P_{i}+\alpha_{i}\left(P_{i}\right)\right) \\
& =-\inf _{P_{i} \in \mathcal{P}_{i}}\left(\int X_{+} d \bigvee P_{i}-\int X_{-} d \wedge P_{i}+\sum_{i} \alpha_{i}\left(P_{i}\right)\right) \\
& =\sup _{P_{i} \in \mathcal{P}_{i}}\left(\int X_{-} d \bigwedge P_{i}-\int X_{+} d \bigvee P_{i}-\sum_{i} \alpha_{i}\left(P_{i}\right)\right) .
\end{aligned}
$$

As consequence of Theorem 3.5 we obtain a further characterization of the Pareto equilibrium condition (E) and under condition (E) the identity of $\widehat{\varrho}$ and $\varrho_{*}$. Therefore, the representation of (3.6) holds true for $\widehat{\varrho}$ under condition (E).

Proposition 3.6 Let $\varrho_{i}$ be convex risk measures and let $\varrho_{*}$ denote the admissible infimal convolution, then it holds:

$$
\begin{aligned}
\varrho_{*} \text { is a convex risk measure } & \Leftrightarrow \varrho_{*}(1)=\varrho_{*}(0)-1 \\
& \Leftrightarrow \text { The equilibrium condition }(\mathrm{E}) \text { holds. }
\end{aligned}
$$

$\operatorname{Under}(\mathrm{E})$ we have $\varrho_{*}=\widehat{\varrho}$.

Proof: The first equivalence is obvious since the condition $\varrho_{*}(1)=\varrho_{*}(0)-1$ implies translation invariance of $\varrho_{*}$. By Theorem 3.5

$$
\begin{aligned}
\varrho_{*}(1) & =\sup \left\{-\left|\bigvee_{j} P_{j}\right|-\sum_{j} \alpha_{j}\left(P_{j}\right) ; \quad P_{j} \in \mathcal{P}_{j}\right\} \\
& =-\inf \left\{\left|\bigvee_{j} P_{j}\right|+\sum_{j} \alpha_{j}\left(P_{j}\right) ; \quad P_{j} \in \mathcal{P}_{j}\right\} .
\end{aligned}
$$

Thus $\varrho_{*}(1)=\varrho_{*}(0)-1$ if and only if there exists a common scenario measure $Q \in$ $\bigcap_{i=1}^{n} \mathcal{P}_{i}$ which by Proposition 3.3 is equivalent to the equilibrium condition (E).

Remark 3.7 The condition $\varrho_{*}(1)=\varrho_{*}(0)-1$ has the following interpretation. The traders in the market try to allocate their risk in the best possible way which leads to a risk $\varrho_{*}(1) \leq \varrho_{*}(0)-1$ for any risk measures $\varrho_{i}$. On the other hand from a regulatory point of 
view the risk measures should be chosen by the traders in a most cautious way in order not to underestimate the whole risk. This game theoretic consideration suggests that in order to obtain that the optimal admissible total risk is reasonable i.e. in our context is a convex risk measure one might expect that the condition $\varrho_{*}(1)=\varrho_{*}(0)-1$ should hold. This idea is confirmed by Proposition 3.6.

$\varrho_{*}$ is a convex monotone risk functional - in particular $\varrho_{*}(0)=\sum_{i=1}^{n} \varrho_{i}(0)-$ but $\varrho_{*}$ is not translation invariant in general. From the definition of $\varrho_{*}$ we obtain

$$
\begin{aligned}
\varrho_{*}(1) & =\inf \left\{\sum_{i=1}^{n} \varrho_{i}\left(X_{i}\right) ; 0 \leq X_{i}, \sum_{i=1}^{n} X_{i}=1\right\} \\
& \leq \varrho_{1}(1)+\sum_{i=2}^{n} \varrho_{i}(0)=\varrho_{*}(0)-1 .
\end{aligned}
$$

As we have seen the modified allocation problem given by $\varrho_{*}$ leads to a senseful version of the allocation problem also when (E) does not hold. We finally get by a modification of $\varrho_{*}$ a convex risk measure $\widehat{\varrho}_{*}$ which we call convex admissible infimal convolution.

Definition 3.8 (Convex admissible infimal convolution) We define the convex admissible infimal convolution risk measure $\widehat{\varrho}_{*}$ by

$$
\begin{aligned}
\widehat{\varrho}_{*}(X) & :=\inf \{m \in \mathbb{R} ; \quad X+m \in \mathcal{A}\} \\
& =\inf \left\{m \in \mathbb{R} ; \quad \varrho_{*}(X+m) \leq 0\right\}
\end{aligned}
$$

where $\mathcal{A}=\mathcal{A}_{\varrho_{*}}=\left\{X ; \varrho_{*}(X) \leq 0\right\}$.

From the definition $\widehat{\varrho}_{*}$ is a convex risk measure with $\mathcal{A}_{\widehat{\varrho}_{*}} \supset \mathcal{A}_{\varrho_{*}}=\mathcal{A}$ and

$$
\widehat{\varrho}_{*} \leq \varrho_{*} .
$$

Theorem 3.9 Let $\varrho_{1}, \ldots, \varrho_{n}$ be convex risk measures, then:

1) $\widehat{\varrho}_{*}$ is the largest convex risk measure $\varrho$ with $\varrho \leq \varrho_{*}$.

2) Under condition (E) holds

$$
\widehat{\varrho}_{*}=\widehat{\varrho}
$$

\section{Proof:}

1) This follows from the definition of $\widehat{\varrho}_{*}$.

2) Under condition (E) $\varrho_{*}$ is a convex risk measure by Proposition 3.6. Therefore $\widehat{\varrho}_{*}=\varrho_{*}=\widehat{\varrho}$ by 1$)$.

\section{Risk allocation with cautious risk attitude}

In this section we take an opposite regulatory point of view on risk allocation corresponding to a cautious risk attitude. The natural allocation problem then is given by the 
supremal convolution

$$
\widehat{\tau}(X):=\sup \left\{\sum_{i=1}^{n} \varrho_{i}\left(X_{i}\right) ; \sum_{i=1}^{n} X_{i}=X\right\},
$$

where $\varrho_{i}$ are convex risk measures. It turns out however, that except in the trivial situation where $\mathcal{P}_{1}=\mathcal{P}_{2}=\cdots=\mathcal{P}_{n}=\left\{P_{1}\right\}$ we do not get by (4.1) a convex risk measure.

As in Section 3 it seems natural to restrict the class of decompositions. Let

$$
A(X):=\left\{\left(X_{i}\right)=\left(\varphi_{i} X\right) ; \quad 0 \leq \varphi_{i} \leq 1, \sum_{i=1}^{n} \varphi_{i}=1\right\}
$$

denote the class of admissible decompositions and define the value of the allocation problem with cautious risk attitude which we call the admissible supremal convolution by

$$
\tau^{*}(X):=\sup \left\{\sum_{i=1}^{n} \varrho_{i}\left(X_{i}\right) ; \quad\left(X_{i}\right) \in A(X)\right\} .
$$

$\tau^{*}$ is a convex monotone risk functional (with $\tau^{*}(0)=\sum_{i=1}^{n} \varrho_{i}(0)$ ) but $\tau^{*}$ is not translation invariant in general. Obviously

$$
\tau^{*} \leq \widehat{\tau}
$$

As in Theorem 3.5, $\tau^{*}$ can be calculated explicitly in terms of the representation scenarios $\mathcal{P}_{i}$ of $\varrho_{i}$.

Theorem 4.1 Let $\varrho_{i}$ be convex risk measures with penalty functions $\alpha_{i}$ and $\tau^{*}$ the corresponding admissible supremal convolution. Let $\mathcal{P}_{i}:=\left\{Q \in \mathrm{ba}(P): \alpha_{i}(Q)<\infty\right\}$. Then

$$
\tau^{*}(X)=\sup \left\{\int X_{-} d \bigvee_{i} P_{i}-\int X_{+} d \bigwedge_{i} P_{i}-\sum_{i} \alpha_{i}\left(P_{i}\right) ; \quad P_{i} \in \mathcal{P}_{i}, 1 \leq i \leq n\right\} .
$$

Proof: As in the proof of Theorem 3.5 we obtain

$$
\begin{aligned}
\tau^{*}(X) & =\sup _{\left(\varphi_{i}\right)} \sum_{i} \varrho_{i}\left(\varphi_{i} X\right) \\
& =\sup _{\left(\varphi_{i}\right)} \sum_{i} \sup _{P_{i} \in \mathcal{P}_{i}}\left(E_{P_{i}} \varphi_{i} Y-\alpha_{i}\left(P_{i}\right)\right), \text { with } Y:=-X \\
& =\sup _{\left(P_{i}\right) \in\left(\mathcal{P}_{i}\right)} \sup _{\left(\varphi_{i}\right)} \sum_{i}\left(\int \varphi_{i} Y d P_{i}-\alpha_{i}\left(P_{i}\right)\right) \\
& =\sup _{\left(P_{i}\right)}\left(\int Y_{+} d \bigvee_{i} P_{i}-\int Y_{-} d \bigwedge_{i} P_{i}-\sum_{i} \alpha_{i}\left(P_{i}\right)\right) \\
& =\sup _{\left(P_{i}\right)}\left(\int X_{-} d \bigvee_{i} P_{i}-\int X_{+} d \bigwedge_{i} P_{i}-\sum_{i} \alpha_{i}\left(P_{i}\right)\right) .
\end{aligned}
$$


Remark 4.2 As consequence of Theorem 4.1 we see that

$$
\tau^{*}(1)=-\inf \left\{\left|\bigwedge P_{i}\right|+\sum \alpha_{i}\left(P_{i}\right) ; \quad P_{i} \in \mathcal{P}_{i}\right\}
$$

and thus

$$
\begin{aligned}
\tau^{*}(1)=\sum \varrho_{i}(0)-1 & \Leftrightarrow\left|\bigwedge P_{i}\right|=1, \quad \forall P_{i} \in \mathcal{P}_{i} \\
& \Leftrightarrow \mathcal{P}_{1}=\mathcal{P}_{2}=\cdots=\mathcal{P}_{n} \text { and }\left|\mathcal{P}_{i}\right|=1 .
\end{aligned}
$$

As in Section 3 we obtain from the modified allocation problem a meaningful convex risk measure.

We define the convex admissible supremal convolution risk measure $\widehat{\tau}^{*}$ by

$$
\widehat{\tau}^{*}(X)=\inf \left\{m \in \mathbb{R} ; \quad X+m \in \mathcal{A}_{\tau^{*}}\right\}=\inf \left\{m \in \mathbb{R} ; \quad \tau^{*}(X+m) \leq 0\right\},
$$

where $\mathcal{A}_{\tau^{*}}=\left\{X \in L^{\infty}(P) ; \tau^{*}(X) \leq 0\right\}$. Then we obtain

Proposition 4.3 The convex admissible supremal convolution risk measure $\widehat{\tau}^{*}$ is the largest coherent risk measure @ such that

$$
\varrho_{i} \leq \varrho \leq \tau^{*} \quad \text { for } 1 \leq i \leq n
$$

The admissibility of a decomposition seems to be a natural postulate. Thus the admissible supremal convolution risk measure offers a solution to the risk measure problem for markets under a cautious attitude towards risk.

\section{References}

Barrieu, P. and N. El Karoui (2002a). Optimal design of derivatives in illiquid markets. Quantitative Finance 2, 1-8.

Barrieu, P. and N. El Karoui (2002b). Reinsuring climatic risk using optimally designed weather bonds. Genova Papers, Risk and Insurance Theory 27, 87-113.

Barrieu, P. and N. El Karoui (2003). Optimal derivatives design and diversification in financial markets with non-tradable risk. Preprint.

Barrieu, P. and N. El Karoui (2004). Optimal derivatives design under dynamic risk measures. In G. Yin et al. (Eds.), Mathematics of Finance, Volume 351 of Contemporary Mathematics, Summer Research Conference on Mathematics on Finance, June 22-26, 2003, in Snowbird, Utah, pp. 13-25. AMS.

Barrieu, P. and N. El Karoui (2005). Inf-convolution of risk measures and optimal risk transfer. Finance and Stochastics 9, 269-298. 
Borch, K. (1960a). Reciprocal reinsurance treaties. ASTIN Bulletin 1, 170-191.

Borch, K. (1960b). The safety loading of reinsurance premiums. Skand. Aktuarietidskr. 1, $163-184$.

Borch, K. (1962). Equilibrium in a reinsurance market. Econometrica 30, 424-444.

Borch, K. (1968). General equilibrium in the economics of uncertainty. In K. H. Borch and J. Mossin (Eds.), Risk and Uncertainty, Volume 30, pp. 247-264. Macmillan.

Bühlmann, H. and W. S. Jewell (1979). Optimal risk exchanges. ASTIN Bulletin 10, 243-263.

Burgert, C. and L. Rüschendorf (2004). Consistent risk measures for portfolio vectors. To appear in Insurance and Mathematics 2005.

Burgert, C. and L. Rüschendorf (2005). Allocations of risks and equilibrium in markets with finitely many traders. Preprint, University Freiburg.

Carlier, G. and R.-A. Dana (2003). Core of convex distortions of probability. Journal of Econometric Theory 13, 199-222.

Chateauneuf, A., R.-A. Dana, and J.-M. Tallon (2000). Optimal risk-sharing rules and equilibria with Choquet-expected-utility. Journal of Mathematical Economics 34, 191-214.

Chavallier, E. and H. H. Müller (1994). Risk allocation in capital markets: Portfolio insurance tactical asset allocation and collar strategies. ASTIN Bulletin 24, 5-18.

Dana, R.-A. (2005). A presentation result for concave Schur concave functions. Math. Finance 15, 613-634.

Dana, R.-A. and I. Meilijson (2003). Modelling agents' preferences in complete markets by second order stochastic dominance. Technical Report 0333, Cahier du Ceremade.

Dana, R.-A. and M. Scarsini (2005). Optimal risk sharing with background risk. Preprint.

Delbaen, F. (2002). Coherent risk measures on general probability spaces. In K. Sandmann et al. (Eds.), Advances in Finance and Stochastics. Essays in Honour of Dieter Sondermann, pp. 1-37. Springer.

Denault, M. (2001). Coherent allocation of risk capital. Journal of Risk 4, 1-34.

Ferguson, T. (1967). Mathematical Statistics. A Decision Theoretic Approach. Academic Press.

Föllmer, H. and A. Schied (2004). Stochastic Finance (2nd edn.). de Gruyter.

Fréchet, M. (1940). Les probabilités associées à un système d'événements compatibles et dépendants; I. Événements en nombre fini fixe, Volume 859 of Actual. sci. industr.

Gerber, H. (1979). An Introduction to Mathematical Risk Theory, Volume 8 of Huebner Foundation Monograph. Wharton School, University of Pennsylvania. 
Gerber, H. U. (1978). Pareto-optimal risk exchanges and related decision problems. Astin Bulletin 10(1), 25-33.

Hardy, G. H., J. E. Littlewood, and G. Pólya (1952). Inequalities (1934, 1st edn.). Cambridge University Press.

Heath, D. and H. Ku (2004). Pareto equilibria with coherent measures of risk. Mathematical Finance 14, 163-172.

Hoeffding, W. (1940). Maßstabinvariante Korrelationstheorie. Schriften des Mathematischen Instituts und des Instituts für Angewandte Mathematik der Universität Berlin 5, pp. 181-233 [Reprinted as: Scale-invariant correlation theory. In: Fischer, N. I. and Sen, P. K. (Eds.) (1994). The Collected Works of Wassily Hoeffding, pp. 55-107. Springer.].

Jouini, E. and H. Kallal (2000). Efficient trading strategies in the presence of market frictions. Review of Financial Studies 14, 343-369.

Jouini, E., W. Schachermayer, and N. Touzi (2005). Law invariant risk measures have the Fatou property. Preprint.

Kaas, R., M. J. Goovaerts, J. Dhaene, and M. Denuit (2001). Modern Actuarial Risk Theory, p. 328. Kluwer Academic Publishers.

Landsberger, M. and I. Meilijson (1994). Comonotone allocations, Bickel-Lehman dispersion and the Arrow-Pratt measure of risk aversion. Annals of Operations Research 52, 97-106.

Leland, H. E. (1980). Who should buy portfolio insurance? Journal of Finance 35(2), 581-596.

Lemaire, J. (1977). Echange de risques entre assureurs et théorie des jeux. ASTIN Bulletin 9, 155-179.

Müller, D. W. (1971). Statistische Entscheidungstheorie. Lecture Notes, University of Göttingen.

Rao, K. and M. Rao (1983). Theory of Charges. A study of finitely additive measures. Academic Press.

Rüschendorf, L. (1981). Stochastically ordered distributions and monotonicity of the OC-function of sequential probability ratio tests. Math. Operationsforschung. Ser. Statistics 12, 327-338.

Rüschendorf, L. (2005). Stochastic ordering of risks, influence of dependence and a.s. constructions. In: N. Balakrishnan, I. G. Bairamov, and O. L. Gebizlioglu (Eds.), Advances in Models, Characterizations and Applications, Volume 180 of Statistics: Textbooks and Monographs. CRC Press.

Ryff, J. V. (1970). Measure preserving transformations and rearrangements. J. Math. Anal. and Applications 31, 449-458.

Schied, A. (2004). On the Neyman-Pearson problem for law-invariant risk measures and robust utility functionals. Annals of Applied Probability 14, 1398-1423.

Seal, H. L. (1969). Stochastic Theory of Risk Business. Wiley. 
Wang, S., V. Young, and H. Panjer (1997). Axiomatic characterization of insurance prices. Insur. Math. Econ. 21, 173-183.

Christian Burgert

Department of Mathematical Stochastics

University of Freiburg

Eckerstr. 1

79104 Freiburg

Germany

\author{
Ludger Rüschendorf \\ Department of Mathematical Stochastics \\ University of Freiburg \\ Eckerstr. 1 \\ 79104 Freiburg \\ Germany \\ ruschen@stochastik.uni-freiburg.de
}

